\title{
Application of Electron Counting to Electron Energy-loss Spectroscopy and Implications for Low-Dose Characterization
}

\author{
James L. Hart ${ }^{1}$, Andrew C. Lang ${ }^{1}$, Asher C. Leff ${ }^{1}$, Paolo Longo ${ }^{2}$, Colin Trevor ${ }^{2}$, Ray Twesten ${ }^{2}$, Mitra L. \\ Taheri $^{1}$ \\ 1. Department of Materials Science and Engineering, Drexel University, Philadelphia PA, USA \\ 2. Analytic Projects R\&D, Gatan, Pleasanton CA, USA
}

Electron counting with direct detection (DD) sensors offers large improvements in resolution and signal to noise ratio (SNR) compared to conventional indirect detection (ID) sensors. The benefits offered by DD sensors have yielded remarkable results for low-dose imaging [1]; however, it is unclear how electron counting would affect electron energy-loss spectroscopy (EELS). Here we quantify the performance of electron counting for EELS by comparing the Gatan K2 summit (DD sensor operated in counting mode) and the Gatan US1000FTXP (ID sensor with scintillator/fiber-optic/CCD design). The results indicate DD EELS will offer major advantages for low-dose spectroscopy.

We first determine the noise properties of each sensor for EELS by evaluating the signal to noise ratio (SNR) as a function of primary electron counts (Figure 1). SNR was determined by acquiring spectra from a $\mathrm{SrTiO}_{3}$ sample. The signal (N) was defined as the average number of electron counts between $1300-1400 \mathrm{eV}$, and the noise was determined as the signal standard deviation after background subtraction. Both sensors were operated at $0.5 \mathrm{eV} / \mathrm{channel}$ dispersion, and the ID sensor was operated at its maximum and minimum vertical binning values. The experimental data was analysed using a model which accounts for shot noise smoothing by the detector, electronic read-out noise, Fano noise, and gain noise. The circular markers show experimental data and the solid lines show the modelled data. For low electron counts, the read-out noise of the ID sensor degrades the EELS SNR, while the DD sensor remains shot noise limited even below 1 electron per energy channel. This improvement in SNR will benefit low-dose analysis of beam sensitive specimens where the number of incident electrons must be minimized.

We next determine how electron counting affects energy resolution. Using a Schottky electron source with a FWHM of $0.6 \mathrm{eV}$, we acquire a zero loss peak (ZLP) with the DD sensor at a dispersion of 0.5 eV/channel. For comparison, ZLPs were acquired the ID sensor at various dispersions. The modulation transfer function (MTF) - representing the system's amplitude response to sinusoidal signals of varying frequency - is then extracted by fitting a sum of Gaussians to the acquired ZLPs, taking the Fourier transform, and then multiplying by a sinc function to account of pixel modulation. Comparing the DD sensor (blue) to the ID sensor at equivalent energy resolution (yellow), there is a clear improvement in MTF. An even larger improvement is observed when comparing DD (blue) to the ID sensor at an equivalent energy field of view of $2 \mathrm{keV}$ (green). EEL spectra from the Ti L edge of a $\mathrm{PbZr}_{0.2} \mathrm{Ti}_{0.8} \mathrm{O}_{3}$ are shown, each acquired with an acquisition time of 0.3 seconds. With the DD sensor at $0.5 \mathrm{eV} / \mathrm{channel}$ dispersion, splitting of the $\mathrm{L}_{2}$ and $\mathrm{L}_{3}$ transitions is observed, as is splitting of $\mathrm{e}_{\mathrm{g}}$ and $\mathrm{t}_{2 \mathrm{~g}}$ transitions. Spectra acquired with the ID sensor at equivalent dispersion (yellow) and energy field of view (green) do not show splitting of $e_{g}$ and $t_{2 g}$ transitions, demonstrating the improved energy resolution offered with DD. At a dispersion of $0.125 \mathrm{eV} /$ channel, the ID sensor shows a slightly improved MTF compared to the DD sensor at $0.5 \mathrm{eV} /$ channel, albeit with $1 / 8$ the energy field of view. Still, the Ti L spectra acquired 
with this dispersion does not show of $\mathrm{e}_{\mathrm{g}}$ and $\mathrm{t}_{2 \mathrm{~g}}$ transitions; noise from the ID sensor shadows the edge fine structure [2].

References:

[1] X. Bai et al, Trends Biochem. Sci. 40, (2015), 49.

[2] The authors acknowledge financial support from NSF MRI award \# 1429661

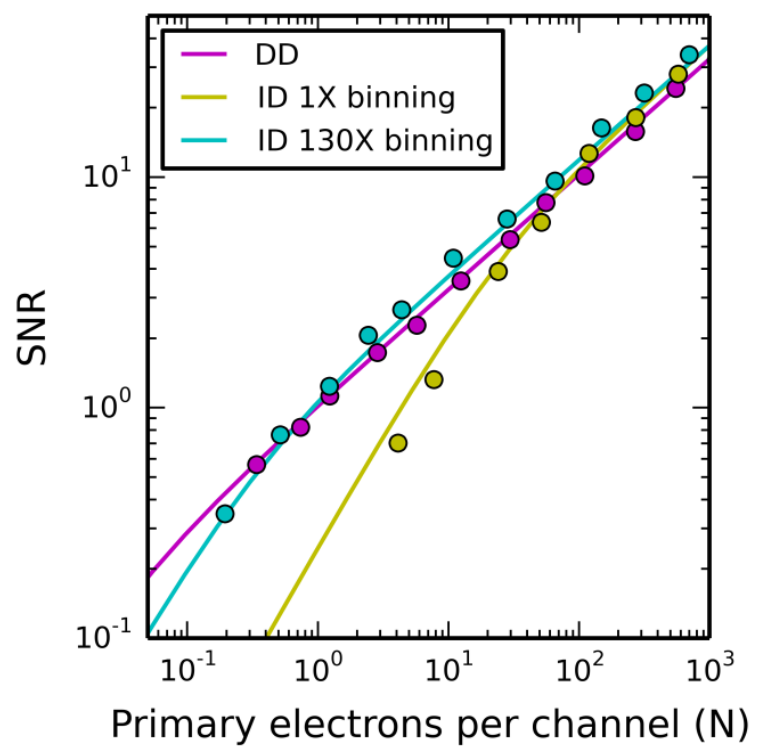

Figure 1. SNR as a function of electron counts per channel for each sensor. The circular markers show experimental data while the solid lines show modeled data.
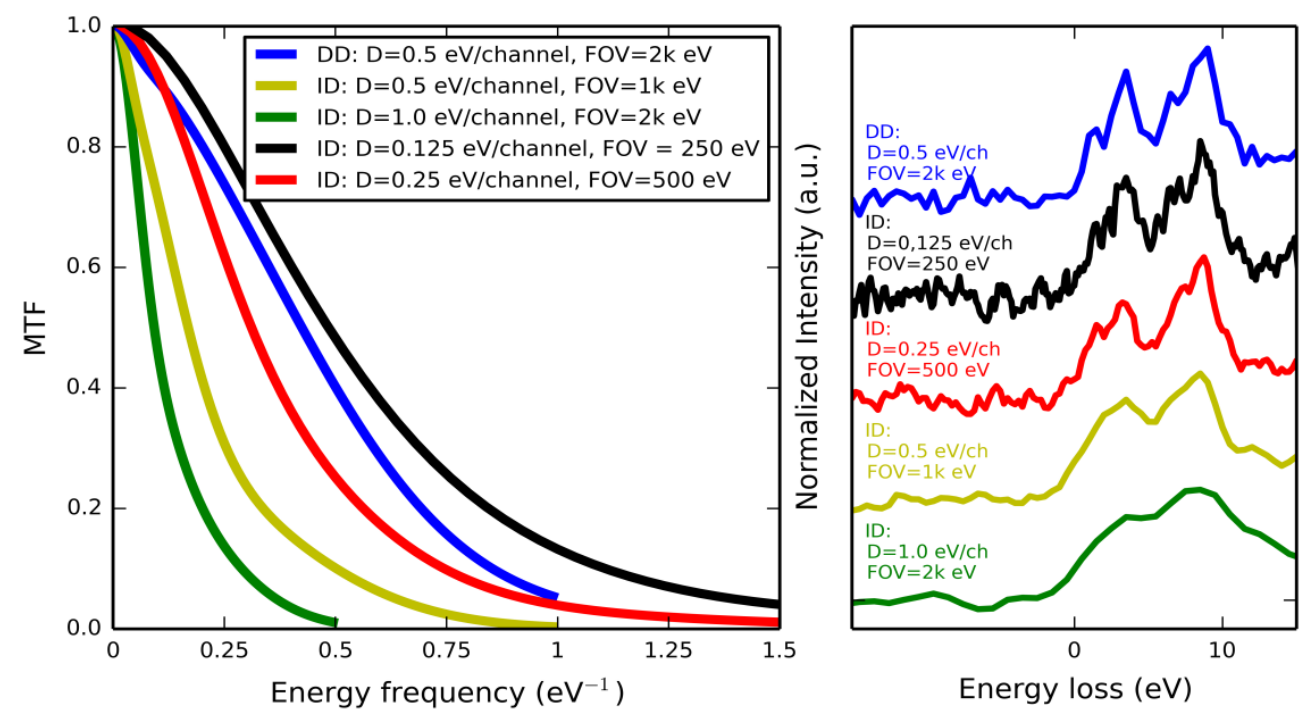

Figure 2. MTFs for each sensor and $\mathrm{Ti} \mathrm{L}$ edge spectra from a $\mathrm{SrTiO}_{3}$ sample after background subtraction. The spectra were normalized according to the integrated $\mathrm{L}_{2,3}$ weight. 